\title{
Contribution of substrate reorganization energies of electron transfer to laccase activity
}

Mehra, Rukmankesh; Kepp, Kasper Planeta

Published in:

Physical Chemistry Chemical Physics

Link to article, DOI:

$10.1039 / \mathrm{c} 9 \mathrm{cp} 01012 \mathrm{~b}$

Publication date:

2019

Document Version

Peer reviewed version

Link back to DTU Orbit

Citation (APA):

Mehra, R., \& Kepp, K. P. (2019). Contribution of substrate reorganization energies of electron transfer to laccase activity. Physical Chemistry Chemical Physics, 21(28), 15805-15814. https://doi.org/10.1039/c9cp01012b

\section{General rights}

Copyright and moral rights for the publications made accessible in the public portal are retained by the authors and/or other copyright owners and it is a condition of accessing publications that users recognise and abide by the legal requirements associated with these rights.

- Users may download and print one copy of any publication from the public portal for the purpose of private study or research.

- You may not further distribute the material or use it for any profit-making activity or commercial gain

- You may freely distribute the URL identifying the publication in the public portal

If you believe that this document breaches copyright please contact us providing details, and we will remove access to the work immediately and investigate your claim 


\title{
Contribution of Substrate Reorganization Energies of Electron Transfer to Laccase Activity
}

\author{
Rukmankesh Mehra and Kasper P. Kepp*
}

Technical University of Denmark, DTU Chemistry, Building 206, 2800 Kgs. Lyngby, Denmark

\section{Corresponding Author}

* Kasper P. Kepp, E-mail: kpj@kemi.dtu.dk

\begin{abstract}
Electron transfer is the most fundamental reaction in chemistry, yet its exact mechanistic details are often complex. Laccases are important electron-transfer enzymes of substantial utility in bleaching, bioremediation, catalytic synthesis, and enzymatic fuel cells. These multi-copper oxidases catalyze the one-electron oxidation of substrates by outer-sphere electron transfer to a copper T1 site, and subsequent intramolecular electron transfer to a tri-nuclear copper site where $\mathrm{O}_{2}$ is reduced to water. Understanding the molecular mechanism of the first, supposedly ratedetermining pure electron transfer step is of major fundamental and technological interest. It is widely thought that the difference in the half potentials of the substrate and the T1 copper enables the powerful electron abstraction from nearby substrates. However, the reorganization energy during electron transfer could also contribute to catalytic turnover. To explore this, we computed the self-exchange reorganization energies of 54 substrates with experimentally known activity or $\mathrm{k}_{\mathrm{cat}}$ data using Density Functional Theory. We show that the energy costs of changing the substrate geometries during electron removal correlate significantly with experimental activity data with a physically meaningful direction of correlation. This means that substrate electronic reorganization, rather than only potential differences, plays a role in the activity of electron transfer proteins such as laccases. This finding is consistent with the Marcus theory and suggests that the first electron transfer step from substrate to $\mathrm{T} 1$ is rate-determining in the real enzymes; the electronic reorganization energies can rationalize "good" vs. "bad" laccase substrates, which has not previously been possible.
\end{abstract}

Keywords: Electron transfer, protein, laccase, reorganization energy, $\mathrm{k}_{\mathrm{cat}}$ 


\section{Introduction}

Laccases are multi-copper oxidases consisting of one mononuclear type 1 (T1) copper (Cu) site, which is responsible for the blue color of these enzymes, and a tri-nuclear cluster containing one $\mathrm{T} 2 \mathrm{Cu}$ ion and two $\mathrm{T} 3 \mathrm{Cu}$ ions. ${ }^{1-3}$ Laccases are found broadly in plants, fungi, bacteria and insects. ${ }^{4,5}$ Fungal laccases can catalyze the oxidation of many diverse substrates including phenols, amines, ketones, ascorbates, phosphates and, arguably in connection with mediators, lignin. ${ }^{6-9}$ Accordingly, laccases have important biotechnological and industrial applications as oxidizing catalysts of organic synthesis, as bleaching agents, in enzymatic fuel cells, photobiocatalysis, and in bioremediation ${ }^{10-14}$.

The structure of the fungal laccases is comprised of three domains (Figure 1a). ${ }^{15,16}$ The T1 $\mathrm{Cu}$ is located near the surface-exposed substrate binding site of domain 3 , and the tri-nuclear cluster is located at the interface between domains 1 and $3 .{ }^{17}$ The $\mathrm{T} 1 \mathrm{Cu}$ is involved in direct one-electron oxidation of the substrates ${ }^{2,15,17}$. As originally seen from the crystal structure of a related multi-copper oxidase, ${ }^{18}$ the $\mathrm{T} 1 \mathrm{Cu}$ then transfers the electron at more than $10 \AA$ distance to the anti-ferromagnetically coupled ${ }^{19}$ oxidized T2/T3 Cu-atoms. ${ }^{2,20,21}$ Four sequential such electron transfers with associated proton transfer finally lead to the reduction of molecular $\mathrm{O}_{2}$ to water at the T2/T3 site. ${ }^{2,20}$ The complex electron transfer activity, even before considering their technological importance, makes laccases of substantial interest in efforts to understand biological electron transfer $17,20,22-25$.

The mechanism of laccases is widely debated, and several of the individual electron and proton transfer steps may contribute to observed activity, depending on oxygen and substrate concentration, proton availability, the presence of redox mediators, salt, small anion inhibitors, and the nature of the substrate ${ }^{6,17}$. The direct one-electron transfer from the substrates to the T1 site is measured in assays and explains the different activity of a given laccase towards different substrates since the protein is unchanged, and it even explains much of the relative activity of different laccases since the subsequent intramolecular electron transfer pathway and T2/T3 electronic structure is highly conserved ${ }^{1,26}$.

The initial electron transfer from the substrate to the T1 site is generally considered the ratelimiting step of turnover ${ }^{1,27,28}$; thus the subsequent electron transfer from T1 to T2/T3 is fast ${ }^{1,17}$, and kinetic analysis of several substrates show that the catalytic efficiency directly relates to the difference between the redox potential of the T1 site and the substrate. ${ }^{29}$ Whereas this first step is a pure outer-sphere electron transfer, subsequent steps involve proton-coupled electron 
transfer $^{1}$. Figure 1d shows the initial electron transfer step studied in this work and the subsequent proton-coupled electron transfer steps in laccase ${ }^{1}$.

Accordingly, the activity of laccases is partly determined by the difference in half reduction potentials of the protein $\mathrm{T} 1$ site and the substrate ${ }^{29}$. Thus, high-potential laccases, with standard half reduction potentials as high as $+0.8 \mathrm{~V}$ vs. the standard hydrogen electrode $(\mathrm{SHE})^{30}$, are particularly proficient electron transfer enzymes in many applications ${ }^{31,32}$. The high half reduction potential of $\mathrm{T} 1$ arises mainly from a reduction in the electron density near the redoxactive $\mathrm{Cu}$ ion, due to removal of a coordinating ligand relative to low-potential laccases ${ }^{31,33}$.

However, most assays of substrate oxidation and associated product formation follow Michaelis-Menten kinetics. The activity thus depends on the substrate accommodation and orientation within the substrate binding site, with $\mathrm{K}_{\mathrm{m}}$ values varying by three orders of magnitude for different laccase substrates ${ }^{23}$. Experimental $K_{m}$ values of fungal laccases correlate with the affinity of the substrates for the T1 binding pocket, estimated using MMGBSA computations ${ }^{34}$. The $\mathrm{K}_{\mathrm{m}}$ values are well-described by molecular-dynamics-derived life-times of the active substrate poses at the T1 site, which again are caused by the substrate affinity for this specific site, rather than the total substrate affinity, which is an ensemble-average of many bound substates. ${ }^{35}$ Thus, the real activity of a laccase depends not only on the redox potential but also on the details of the substrate's ability to fit the $\mathrm{T} 1$ site $^{35}$ and become oxidized by proper alignment, with the electron transfer rate decaying exponentially with the substrate donor atom distance from the $\mathrm{T} 1 \mathrm{Cu}^{23}$.

Furthermore, the observed catalytic activity could also depend on the electronic reorganization energy $(\lambda)$ of Marcus theory, i.e. the change in electronic energy caused by a change in nuclear geometry. The total $\lambda$ is the sum of the protein and the substrate contributions and further has an inner-sphere and an outer-sphere component, the later being the long-range solvent and protein response to the electron transfer (ET) process. The protein component of the reorganization energy has been previously explored in detail and we thus understand the copper reorganization energies fairly well. ${ }^{17,36,37}$ The role of protein $\lambda$ in the inhibition of laccases by small halides has also been studied. ${ }^{38}$ Galli et al. previously studied the oxidation by laccases from Trametes villosa, T. versicolor and a T. versicolor D206A mutant of six phenolic substrates and in this process accounted for the importance of $\lambda .^{22}$ The $\mathrm{T} 1 \lambda$ is $\sim 0.5-0.8 \mathrm{eV}$ and similar for $\mathrm{T} 1$ sites of different proteins with the same coordination geometry ${ }^{39-41}$, as $\lambda$ is dominated by the inner-sphere component of the first coordination sphere ${ }^{17,42,43}$. Thus, any contribution of $\lambda$ to relative experimental activities should arise via the substrate's self-exchange $\lambda$. 
To explore this hypothesis, we studied substrate total $\lambda$ for two datasets comprising total 54 substrates using Density Functional Theory (DFT) and compared to experimental activity data, to see if the contribution from substrate $\lambda$ has been overlooked, is important, and can be modeled well. The large datasets by Sulistyaningdyah et al. ${ }^{44}$ and $\mathrm{Xu}^{29}$ were used. Sulistyaningdyah et al. reported the relative activity of laccases from T. versicolor, Myrothecium verrucaria and Trametes sp. Ha-1 toward phenols and anilines. ${ }^{44} \mathrm{Xu}$ analyzed the oxidation of phenols, anilines and benzenethiols by fungal laccases and studied the correlation between the activity and redox potentials. ${ }^{29}$ We studied the activities of $T$. versicolor laccase reported by Sulistyaningdyah et al. ${ }^{44}$ (dataset $\mathrm{A}-20$ substrates) and the $\mathrm{k}_{\mathrm{cat}}$ values of $T$. villosa laccase reported by $\mathrm{Xu}^{29}$ (dataset B - 34 substrates).

We find that the substrates displaying small structural changes and hence low $\lambda$ tend to have higher catalytic activities and vice versa. For one dataset (dataset A), the relationship is statistically significant at $99 \%$ confidence $(\mathrm{p}<0.01)$, whereas for the larger more diverse dataset (dataset B), it is significant at the 95\% confidence level after leaving out two of the 34 data points. This shows that i) the used four-point model $^{45}$ can describe the relative activities of electron-transfer proteins such as laccases since it correlates with experimental data for a sizable dataset; ii) the first electron transfer step is rate-determining for the laccase assays typically studied; iii) the reorganization energy contributes to observed laccase activities. These findings should be of both theoretical value to understand laccase catalysis, and to understand the modulation (by e.g. chemical pretreatment) of substrates such as lignin towards higher catalytic activity by lowering the reorganization energy and hence barrier for the rate-determining step. 


\section{Computational Methods}

\section{Studied datasets and substrate models}

The two datasets (dataset A and B) were studied in the present work (Table 1). Dataset A consists of 20 laccase substrates for which the relative activity was reported for T. versicolor laccase ${ }^{44}$, of the general type shown in Figure 1b. Dataset B comprises 34 compounds with reported $\mathrm{k}_{\text {cat }}$ values for T. villosa laccase ${ }^{29}$, of the type shown in Figure 1c.

To ensure a consistent, and computable dataset, two dataset A compounds $n$-propylgallate and $p$-phenylenediamine were excluded as their activities were not reported for $T$. versicolor laccase, and the very large and distinct ABTS, which served as standard, was not studied. In addition, the activity was not reported for one compound (37) of dataset $\mathrm{B}^{29}$ and thus not studied, and two other compounds of dataset B (18 and 19) were tagged with lysozyme, and these compounds were thus not included in the study as they cannot be realistically modeled. The laccases have different activities at different $\mathrm{pH}$, but at any particular $\mathrm{pH}$, the contribution of the protein to the activity is constant, and any difference in activity of two substrates arises mainly from the substrate's contribution if the effects are not correlated by e.g. ligand-induced protonation of the protein. To account for the effect of $\mathrm{pH}$ on activity, we studied the two datasets at the different protonation states prepared at experimental $\mathrm{pH}$, i.e. $\mathrm{pH} 9$ for dataset $\mathrm{A}$ and $\mathrm{pH} 5$ for dataset $\mathrm{B}$. The experimental activities and $\mathrm{k}_{\mathrm{cat}}$ values were converted to the $\log _{10}$ scale in order to enable direct comparison to the computed energies. The structural formula and experimental activity parameters of all studied compounds are shown in Supplementary Table S1.

The structures of the normal-charged substrates (referred to as "x") were initially prepared using the 2D-sketcher of Maestro, and the geometries and protonation states used as input for the DFT geometry optimization were prepared using the OPLS3 force field and Ionizer in LigPrep. $^{46}$ 
Table 1. Datasets studies in the present work.

\begin{tabular}{|l|l|l|l|l|l|}
\hline Dataset & $\begin{array}{l}\text { Number of } \\
\text { substrates }\end{array}$ & Activity type & pH & Type of compounds & Reference \\
\hline A & 20 & $\begin{array}{l}\text { Relative activity with } \\
\text { respect to phenol }\end{array}$ & 9.0 & $\begin{array}{l}\text { Phenols, anilines, } \\
\text { thiols, carboxylic } \\
\text { acids }\end{array}$ & 44 \\
\hline B & 34 & $\mathrm{k}_{\text {cat }}$ & 5.0 & $\begin{array}{l}\text { Phenols, amines, } \\
\text { nitros, aldehydes, } \\
\text { thiols and carboxylic } \\
\text { acids }\end{array}$ & 29 \\
\hline
\end{tabular}

(a)

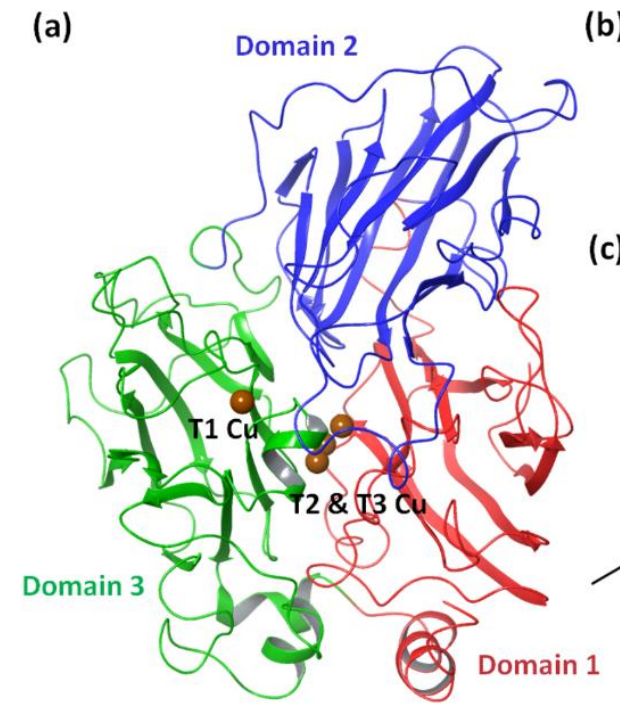

(b)<smiles>Oc1ccccc1</smiles>

(c)<smiles>Oc1ccccc1</smiles>

I<smiles>Nc1ccccc1</smiles>

II

$\mathrm{HO}$<smiles>COc1ccccc1O</smiles>

II<smiles>[R]c1ccccc1</smiles>

IV (d) $\mathrm{T} 1 \mathrm{Cu}(\mathrm{II})$

T3 $\alpha \mathrm{Cu}(\mathrm{II}), \beta \mathrm{Cu}(\mathrm{II})$

$\mathrm{T} 2 \mathrm{Cu}(\mathrm{II})$

Native intermediate

$$
\hat{r}_{\mathrm{s}^{+}}^{\mathrm{s}}
$$

$\mathrm{T} 1 \mathrm{Cu}(\mathrm{I})$

T3 $\alpha \mathrm{Cu}(\mathrm{II}), \beta \mathrm{Cu}(\mathrm{II})$

$\mathrm{T} 2 \mathrm{Cu}(\mathrm{II})$

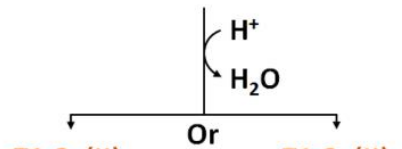

$\mathrm{T} 1 \mathrm{Cu}(\mathrm{II}) \quad \mathrm{T} 1 \mathrm{Cu}(\mathrm{II})$

$\mathrm{T} 3 \alpha \mathrm{Cu}(\mathrm{II}), \beta \mathrm{Cu}(\mathrm{II}) \quad \mathrm{T} 3 \alpha \mathrm{Cu}(\mathrm{II}), \beta \mathrm{Cu}(\mathrm{II})$ $\mathrm{T} 2 \mathrm{Cu}(\mathrm{I})$ $\mathrm{T} 2 \mathrm{Cu}(\mathrm{II})$

Figure 1. (a) Structure of laccase from T. versicolor (PDB code: 1GYC). (b) Dataset A mainly consists of similar-sized phenols (15 compounds; scaffold I) with substituted $\mathrm{OH}, \mathrm{Cl}, \mathrm{COOH}$ and $\mathrm{OCH}_{3}$ groups. It also includes five substituted anilines (scaffold II). (c) Dataset B mainly consists of phenols (scaffolds I, II, III) with substituents varying from carboxyl and amine to aldehyde, thiol and nitro groups, or fragments containing these groups, but also includes seven non-phenols with substitutions on benzene including $\mathrm{NH}_{2}, \mathrm{SH}, \mathrm{COOH}, \mathrm{F}$ and $\mathrm{OCH}_{3}$ groups (scaffold IV, where $\mathrm{R}$ is $\mathrm{NH}_{2}, \mathrm{SH}$, or $\mathrm{COOH}$ ). (d) Initial rate-limiting outer-sphere electron transfer step of substrate (S) oxidation vs. subsequent proton-coupled electron transfer steps, based on Figure 35 of the review by Solomon et al. ${ }^{1}$ 


\section{DFT Computations}

All quantum mechanical calculations were performed using the Jaguar software, version 9.8 (release 11). ${ }^{47,48}$ Geometry optimization was performed using redundant internal coordinates with default convergence criteria and the initial Hessian determined using a Schlegel guess as implemented in Jaguar. DIIS and GDIIS methods were used for convergence. ${ }^{49,50}$ Harmonic vibrational frequencies and the thermochemical corrections were calculated using the default setup at 1 atmosphere pressure and $\mathrm{T}=298.15 \mathrm{~K} .{ }^{47}$

To investigate the sensitivity of our results to the use of computational method, we performed all computations completely in parallel using three density functionals with different design principles: The widely used hybrid GGA functional B3LYP ${ }^{51-53}$, the meta hybrid functional $\mathrm{M}^{54}{ }^{54}$, and the non-hybrid functional PBE. ${ }^{55}$ We suspected that the difference in Hartree-Fock exchange (20\% in B3LYP, 27\% in M06, 0\% in PBE) could, among other features, affect the ability of DFT to describe the experimental trend chemistry; as shown below it does not, due to the high level of error cancellation in computed $\lambda$, making the results very robust to method choice compared to many other computed chemical energies.

From previous work ${ }^{56,57}$, we know that trends in reorganization energies, which are required for correlation to relative activity data, require a polarized double-zeta basis but are not as sensitive to basis sets as other chemical energies because of the large cancellation of errors in the four-point calculation ${ }^{45,58}$. The geometries need to be well-described, in particular by also including polarization functions on hydrogen atoms. It is considered more important to compute the single point energies at the exact same level of theory as the geometry optimized ground states in order to estimate the vertical ionization energies and electron affinities correctly, otherwise a shift of the potential energy curve would occur ${ }^{42}$. This study includes 108 geometry optimizations of compounds of very variable size, and thus we used the $6-31 \mathrm{G}^{* *}$ basis set that includes polarization functions on all atoms, estimating that basis set errors, which tend to cancel in the four energies once combined, is of the order of $1-3 \mathrm{kcal} / \mathrm{mol}$ and will thus not affect the trends described below in the results section, which are governed by changes up to typically 20$30 \mathrm{kcal} / \mathrm{mol}$. We thus considered it more important to study a large dataset than to model a small amount of compounds at higher accuracy.

In total, the 54 compounds at their normal protonation states (at $\mathrm{pH} 9$ for dataset $\mathrm{A}$ and $\mathrm{pH}$ 5 for dataset B) and the corresponding 54 one-electron oxidized systems were geometry 
optimized (108 systems) with each of the three functionals, i.e. 324 geometry optimizations in total. In addition to these computations, 324 additional single-point energies were computed, i.e. the energies of the one-electron oxidized state of each compound at its geometry optimized at normal protonation, $\mathrm{E}_{\mathbf{x}+}(\mathbf{x})$, and the corresponding normal energy (at normal protonation state) evaluated at the geometry of the one-electron oxidized compounds, $E_{\mathbf{x}}\left(\mathbf{x}^{+}\right)$.

The self-exchange substrate reorganization energies were calculated using the 4-point model by Nelsen et al. ${ }^{45}$ as shown in Equation 1:

$$
\lambda=E_{\mathrm{x}}\left(\mathrm{x}^{+}\right)-\mathrm{E}_{\mathrm{x}}(\mathrm{x})+\mathrm{E}_{\mathrm{x}}^{+}(\mathrm{x})-\mathrm{E}_{\mathrm{x}}^{+}\left(\mathrm{x}^{+}\right)
$$

Here, $\mathrm{E}_{\mathrm{x}}\left(\mathrm{x}^{+}\right)$is the single point energy of species $(\mathrm{x})$ on the optimized structure with one less electron $\mathrm{x}^{+}$, and $\mathrm{E}_{\mathrm{x}}(\mathrm{x})$ is the converged energy of the optimized geometry of the state $(\mathrm{x})$. Please note that we computed the substrate reorganization but ignored any protein contribution, which is a constant since the same protein is involved in the assays. We also ignored the heterogeneous outer-sphere contribution to the reorganization energy that is not describable as a dielectric shielding effect. For some substrates, interactions with solvent or protein residues may differ from others in a way that affects structural reorganization, and this effect is not computationally feasible to study with QM/MM and adequate sampling for all substrate-protein-water systems.

However, to understand the likely effects of these long-range components in a computationally cheaper and thus feasible way, we tested the sensitivity of our results to the effect of a solvent model and the thermal chemical corrections by computing the reorganization energies in four ways: Based on the total internal energies corrected for zero-point energies and thermal corrections, based on the total free energies estimated from the vibrational state function and assuming ideal gas entropy, based on the solution-phase electronic energies, and based directly on the gas phase energies as usually applied. The solvent calculations were performed using the Poisson-Boltzmann (PBE) solvation model with water parameters, i.e. a dielectric constant of 80.4 and a probe radius of $1.40 \AA$.

The active poses within the protein require major assumptions in the docking of many of these substrates, with associated changes in the protein structures and large potential errors that will be very difficult to reproduce. Thus, instead we studied the reproducible free substrates and estimated the sensitivity towards conformational changes by docking the six most flexible compounds at $\mathrm{T} 1$ site of the T. versicolor laccase (PDB ID: $1 \mathrm{GYC}^{16}$ ) using SiteMap ${ }^{59}$ and Glide $\mathrm{XP}^{60,61}$ and computed the reorganization energy after geometry optimization from these poses (Supplementary Table S2). While some fall back to the free conformation and produce almost 
identical values for $\lambda$, two compounds produce distinctly different conformations with changes in $\lambda$ up to $4 \mathrm{kcal} / \mathrm{mol}$, which we thus consider the upper limit to conformation-sensitivity in our study of the most flexible systems. This effect importantly does not change any of the trend chemistry, and hence conclusions reported below.

\section{Results and discussion}

\section{Trends in reorganization energy}

We analyzed the correlation between the experimental activities reported for datasets A and B, and the $\lambda$ values for self-exchange electron transfer of the substrates, computed using Equation 1. Considering the constant $\mathrm{T} 1 \lambda$ for high-potential laccases having the same first coordination sphere, we only studied the $\lambda$ contribution of the substrates. We studied the sensitivity of the relationships to the choice of density functional by studying three different functionals, and to the inclusion of vibrational, thermal, and solvent corrections to the energies. The resulting plots using the vibrational corrected energies are shown in Figures $\mathbf{2}$ and $\mathbf{3}$ for datasets $\mathrm{A}$ and B, respectively. Total energies in Hartree are collected in Supplementary Tables S3-S8, and $\lambda$ values are collected in Supplementary Tables S9 and S10. The corresponding plots based on the estimated free energies, solution phase, and gas-phase energies without vibrational corrections are shown in Supplementary Figures S1-S6, with computed energies reported explicitly in Supplementary Tables S11-S28 and the calculated $\lambda$ collected in Supplementary

\section{Tables S29-S34.}

The main result of these computations is that a similar trend between computed $\lambda$ values and experimental data was observed using all four types of energies (see Supplementary data cited above). Accordingly, the solvent contribution and vibrational contribution to the self-exchange $\lambda$ are relatively constant for all substrates such that the correlation with real experimental data is unaffected. There is a sizable contribution of the protein and the solvent to the total $\lambda$ but as one could expect, these contributions are constant for the different substrates oxidized by the same protein. This largely confirms the validity of using the substrate self-exchange reorganization energies specifically. We cannot exclude the possibility of an additional, differential contribution to turnover from outer-sphere substrate reorganization due to explicit substrate-solvent interactions that are not included in the dielectric model and would require very long sampling time with QM/MM or a polarizable force field. Such effects would work to make the substrate 
reorganization energy even more important to real turnover than observed already from the substrate reorganization with the homogeneous dielectric effects added. Accordingly, we discuss below for simplicity only the electronic, vibrational corrected thermal energy estimates of $\lambda$.

\section{Reorganization energy: Effect of different functionals}

Using the widely applied hybrid functional B3LYP, we observed a semi-quantitative correlation between the experimentally reported relative activity and the computed $\lambda$ of dataset A (Figure 2a), a relationship that only improves modestly by removing the largest outlier (Figure $\mathbf{2 b}$ ). Correlation was also seen, after removal of two outliers, between $\mathrm{k}_{\mathrm{cat}}$ and $\lambda$ of dataset B (Figure 3a vs. 3b). However, dataset B was not as well described because of the following reasons: i) the high chemical diversity, ranging from smaller to large compounds; ii) the experimental data were compiled from different sources; iii) the detection limit of the assay causes five compounds to show same small $\mathrm{k}_{\text {cat }}$ (compounds $27, \mathbf{2 8}, \mathbf{2 9}, \mathbf{3 4}$ and $\mathbf{3 5}$, numbering used in original work, with $\left.\mathrm{k}_{\text {cat }} \leq 40 \mathrm{~min}^{-1}\right)$; and iv) \pm 100 standard deviations for the most $\mathrm{k}_{\text {cat }}$ values. Therefore, we expected this dataset to be much harder to model and but included it because it is the largest known laccase substrate dataset from one study. Still, the correlation with 32 out of 34 substrates strongly suggests that the relationship is real for both datasets, as the correlations become significant at the $95 \%$ confidence level $(\mathrm{p}<0.05)$. The authenticity of our finding is further confirmed by the fact that the direction of correlation is physically meaningful, i.e. a larger reorganization energy corresponding to a larger geometry change during substrate oxidation relates to a slower catalytic process (Figures $\mathbf{2}$ and $\mathbf{3}$ ).

To further test the validity of the relationships, we performed the analysis for both datasets completely in parallel using also PBE and M06. The functionals differ substantially in their mathematical design and contain very different Hartree-Fock exchange $(0 \%$ in PBE, $20 \%$ in B3LYP, and 27\% in M06), which is a major contributor to the diverse results commonly found when using $\mathrm{DFT}^{62-64}$.

In dataset A, for B3LYP (Figure 2a), M06 (Figure 2c) and PBE (Figure 2e), generally similar values of $\lambda$ were obtained with typical deviations of a few $\mathrm{kcal} / \mathrm{mol}$ at most, and accordingly, the correlations remain significant. $\mathrm{R}^{2}$ of $0.31,0.35$ and 0.36 were found using B3LYP, M06 and PBE, respectively (Figure 2a, 2c and 2e). When a single compound was left out, the correlations increased further (Figure 2b, 2d and 2f) to 0.37, 0.40 and 0.41 using B3LYP, M06, and PBE, respectively. 
Thus, our results are not very sensitive to the choice of functional. This conclusion is, importantly, specific to the computation of $\lambda$ where four energy evaluations work to cancel the effects of the different functionals, making these computations very robust and useful for the evaluation of trend chemistry vs. experimental data, as seen previously ${ }^{42}$. A similar conclusion applies to dataset B after removal of the difficult outliers (Figure 3). Although the correlation is still worse than for dataset $A$, it is significant at the $95 \%$ confidence level $(p \leq 0.05)$.

This observation suggests that the real catalytic activity of the enzymes depends on the electronic reorganization energies of the substrates during the electron transfer process; the lower the reorganization energy, the higher is the catalytic activity. The results are very robust to method of computation and largely reflect structural changes in the substrates during oxidation.
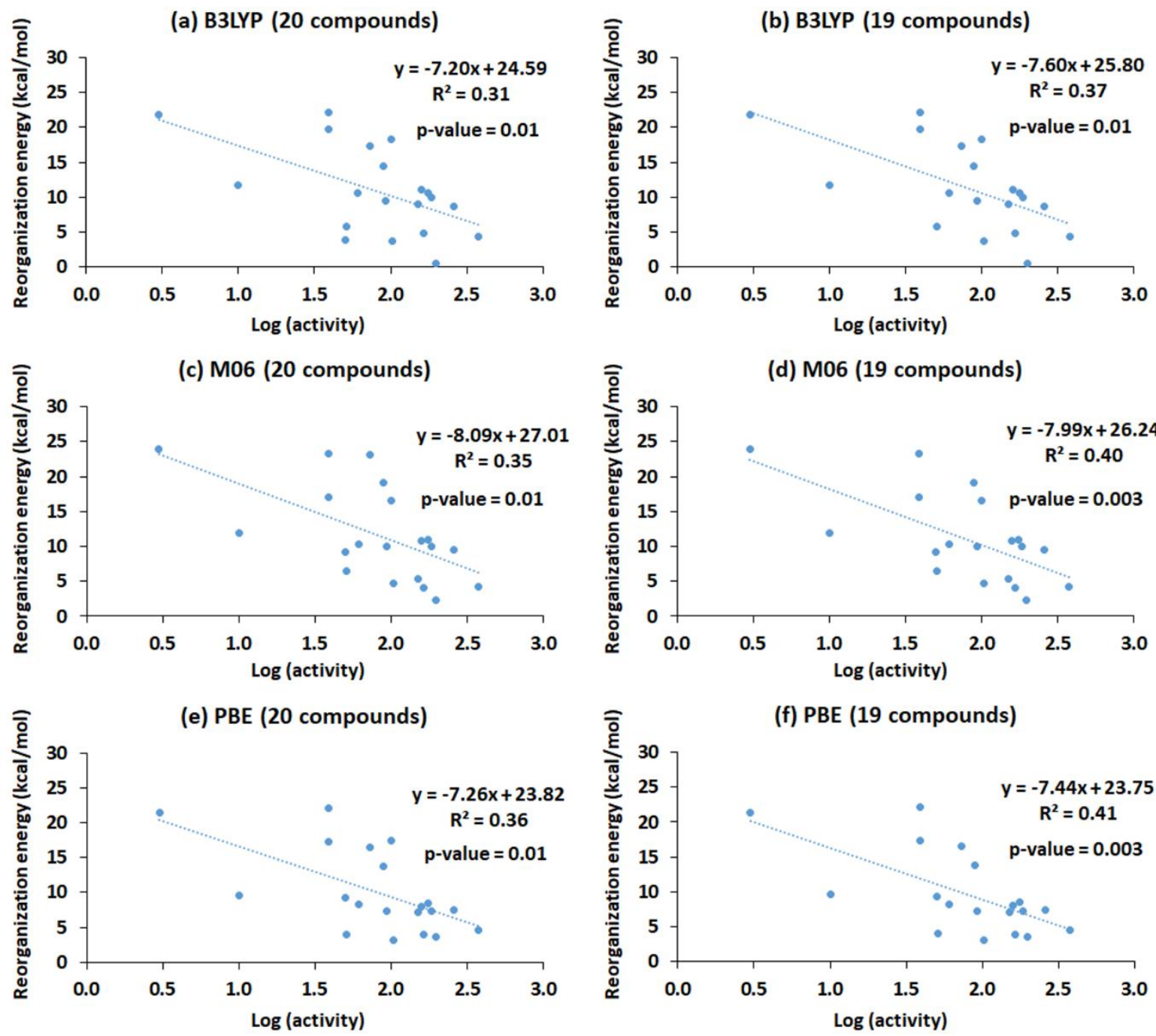

Figure 2. Correlation between experimental log (activity) and computed substrate reorganization energy ( $\mathrm{kcal} / \mathrm{mol}$ ) of dataset A compounds. (a) Correlation using B3LYP. (b) Correlation using 
B3LYP when one compound ( $m$-chlorophenol) was left out. (c) Correlation using M06. (d) Correlation using M06 when one compound (resorcinol) was left out. (e) Correlation using PBE. (f) Correlation using PBE when one compound (phenol) was left out.

As mentioned earlier, dataset B was harder to model because it contains generally more diverse compounds with several compounds assayed below the detection limit, and partly because of compound 13. This compound has the highest molecular weight and includes a total of four methoxy substituents on two phenyl rings (Supplementary Table S2). For PBE, correlation becomes significant (95\% confidence) already after removing only this compound from consideration $\left(\mathrm{R}^{2}=0.13\right.$ and $\left.\mathrm{p}=0.04\right)$ (Supplementary Figures S7). For B3LYP and M06, the correlations were not significant at this confidence level (Supplementary Figures S8 and S9). When the two main outlier compounds were removed from dataset $\mathrm{B}$, the correlations become significant using all the three functionals (Figure $\mathbf{3 b}, \mathbf{d}$ and $\mathbf{f}$ ) with p-values of 0.05 , 0.03 and 0.02 for B3LYP, M06 and PBE, respectively. Though the correlations were low $\left(\mathrm{R}^{2}=\right.$ $0.12-0.17)$, the trend agreement is still significant despite high noise in the experimental data. 

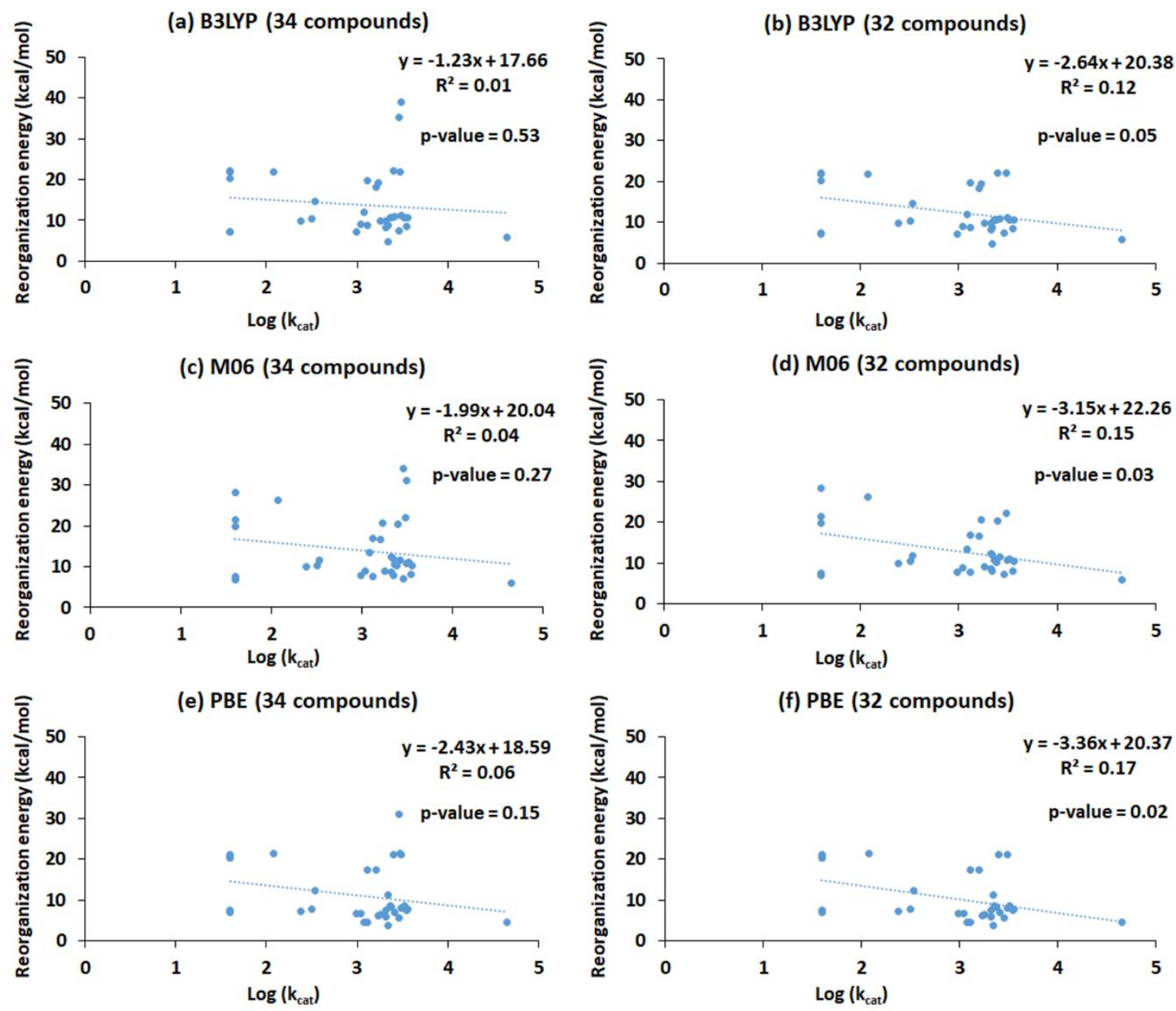

Figure 3. Correlation between experimental $\log \left(\mathrm{k}_{\mathrm{cat}}\right)$ and computed substrate reorganization energy ( $\mathrm{kcal} / \mathrm{mol}$ ) of dataset B compounds. (a) Correlation using B3LYP. (b) Correlation using B3LYP when compounds 13 and 15 were left out. (c) Correlation using M06. (d) Correlation using M06 when compounds $\mathbf{1 3}$ and 15 were left out. (e) Correlation using PBE. (f) Correlation using PBE when compounds 13 and 25 were left out.

In general, PBE showed a slightly better correlation than B3LYP and M06 for both datasets. The fact that better relationships were observed with $\log$ (relative activity) than with $\log \left(\mathrm{k}_{\mathrm{cat}}\right)$ is largely attributable to the noise and detection limit encountered in dataset B. Dataset B (with $\mathrm{k}_{\text {cat }}$ ) is also comparatively large and diverse with phenols, amines, aldehydes, nitro groups, thiol and carboxyl groups, and thus a high correlation was never expected. The largest problem is the detection limit of the assay which makes five compounds having the same reported small $\mathrm{k}_{\text {cat }}$ (compounds 27, 28, 29, 34 and 35, using the original numbering, with $\mathrm{k}_{\text {cat }} \leq 40 \mathrm{~min}^{-1}$ ) as seen in the left side of the panels in Figure 3, although these compounds probably have distinct 
activities. Thus, the limitations in the experimental assay probably explain most of the impaired relationship relative to dataset $\mathrm{A}$. Also, most of the $\mathrm{k}_{\mathrm{cat}}$ values have standard deviations of \pm 100 contributing noise to the fit to the same extent as the estimated $2-3 \mathrm{kcal} / \mathrm{mol}$ computational uncertainty in $\lambda .{ }^{29}$ In the other end of the spectrum, the five most active compounds $(\mathbf{1 0}, \mathbf{1 4}, \mathbf{2 2}$, 23 and 31 with $\mathrm{k}_{\text {cat }}=3100-45000 \mathrm{~min}^{-1}$ ) all have $\lambda$ values that are consistently small (4.5-11.1 $\mathrm{kcal} / \mathrm{mol}$ ) using all three functionals (Supplementary Table S10).

Whereas the diversity of dataset B reduces the correlation, the high significance after outlier removal, the significant correlation for dataset $\mathrm{A}$, and the meaningful and consistently similar direction of correlations using all three functionals suggest that our initial hypothesis is correct, i.e. the experimental activity of laccases is partially determined by the $\lambda$ of the substrates. This means that the catalytic activity is more favored if the electron-donating group of a substrate can easily transfer the electron without changing very much its geometry and associated electronic energy. Figure 4 shows this principle schematically: The change in the substrate geometry changes the energy of the electronic states such that substrates with low $\lambda$ tend to change little compared to the substrates with high $\lambda$ once they are oxidized by the laccase, and this contributes to a faster electron transfer to the $\mathrm{T} 1 \mathrm{Cu}$ site.

\section{Contribution of structural reorganization to laccase activity}

For dataset $\mathrm{A}$, the $\mathrm{x}$ - and $\mathrm{y}$-intercepts of the plots in Figure 2 suggest that $\lambda$ values of 24-26 $\mathrm{kcal} / \mathrm{mol}$ will reduce the observed activity to zero. These intercepts are remarkably similar for all methods studied but differ somewhat between the two datasets. Correspondingly, we find that $\log$ (activity) is 3.2-3.4 when $\lambda$ approaches zero (Supplementary Table S35). This intercept arguably represents the maximal activity of the laccase at zero substrate reorganization for a constant contribution of other effects (reduction potential difference, electron transfer pre-factor, and protein and solvent reorganization energy), with some phenolic compounds already close to achieving this limit.

For dataset $\mathrm{B}$, a physically meaningful inverse dependence of $\lambda$ on $\mathrm{k}_{\text {cat }}$ is also evident (Figure 3). The $\mathrm{x}$ - and $\mathrm{y}$-intercepts suggest $\lambda$ of $20-22 \mathrm{kcal} / \mathrm{mol}$ when $\log \left(\mathrm{k}_{\mathrm{cat}}\right)$ approaches zero, and $\log \left(\mathrm{k}_{\mathrm{cat}}\right)$ of $6.1-7.7$ when $\lambda$ approaches zero (Supplementary Table S36). The similar values of $\mathrm{x}$ - and $\mathrm{y}$-intercepts using all three functionals again suggest a robust real interpretation of the limits of the contribution of substrate reorganization to laccase catalytic activity. The large $\mathrm{k}_{\text {cat }}$ values and the large contribution of $\lambda$ in Figure 3 suggests that substrate reorganization is a 
significant contributor to the experimentally measured turnover and that very high activity can be achieved if one can reduce the substrate's reorganization energy by chemical manipulation. Thus, we suggest that pretreatments used for complex phenolic substrates such as lignin reduce the size, accessibility, and requirements for structural reorganization via $\lambda$.

\section{Good versus bad laccase substrates}

To understand "good" and "bad" laccase substrates, we investigated some of the extreme compounds in both datasets. In dataset A, the most active compound NN-dimethyl- $p$ phenylenediamine (relative activity $=377)$ has a very small substrate $\lambda(4.2-4.6 \mathrm{kcal} / \mathrm{mol}$ for the different functionals) reflecting a very minor change in geometry upon oxidation (Figure 4a). In contrast, the least active compound of this dataset, $p$-hydroxybenzoic acid (relative activity $=3$ ), has a very large $\lambda(21.4-23.9 \mathrm{kcal} / \mathrm{mol})$ (Figure $4 \mathbf{b}$ and Supplementary Table S9). Interestingly, by changing only a single bond from hydrocaffeic acid to caffeic acid (Supplementary Table S1), a major decrease in the relative experimental activity is seen from 185 to 39 . This surprising change is well-explained by an increase in $\lambda$ from 9.9 to $22.1,9.9$ to 23.2 and 7.3 to $22.1 \mathrm{kcal} / \mathrm{mol}$ using B3LYP, M06 and PBE respectively (Supplementary Tables S9), and could probably not have been understood without such computation. As a difficult case, all the four chlorosubstituted phenols show consistently small $\lambda$ values of 3.9-9.9 $\mathrm{kcal} / \mathrm{mol}$, despite a substantial variation in the relative experimental activity (from 50 to 258; Supplementary Table S9). Except from these chlorophenols, the performance is generally robust across the compound classes. 
(a) Substrates with low $\lambda$

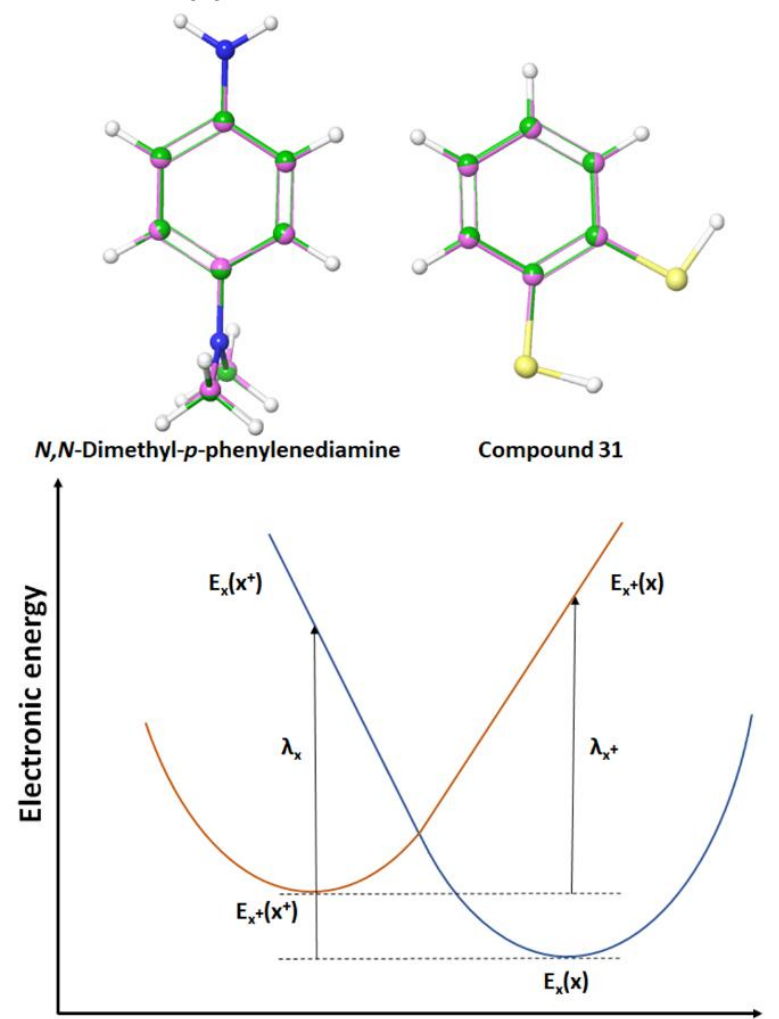

Reaction coordinates (b) Substrates with high $\lambda$

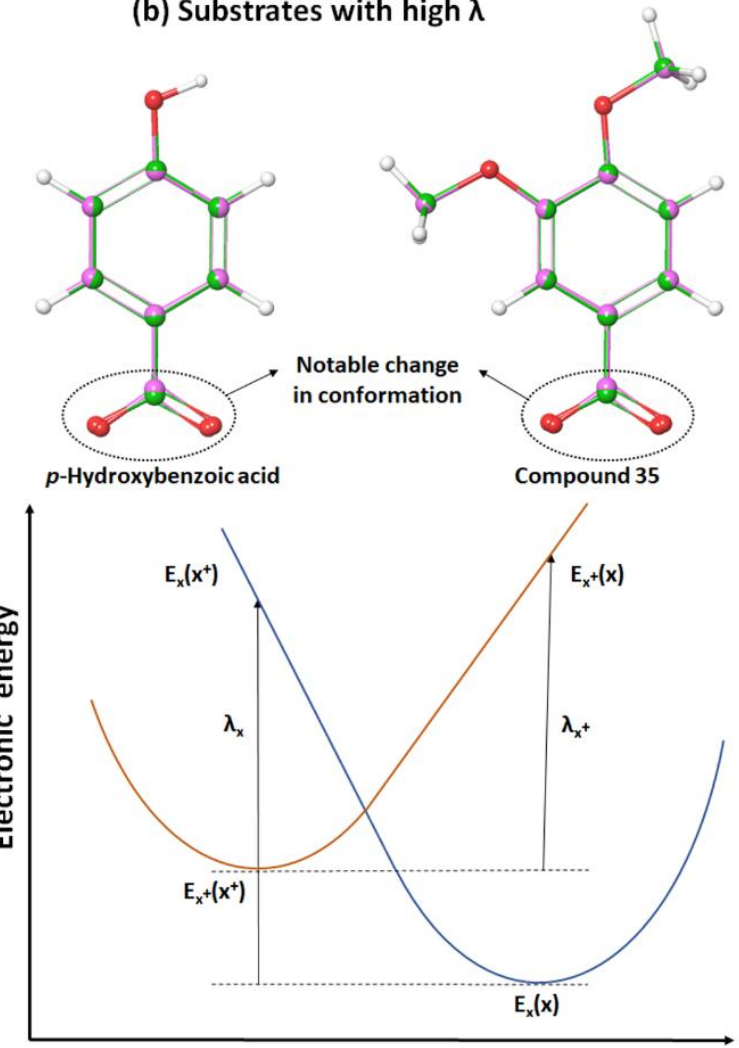

Reaction coordinates

Figure 4. Change in the substrate conformations with change in electronic states and reorganization energies $\left(\lambda=\lambda_{\mathrm{x}}+\lambda_{\mathrm{x}+}\right)$. Representative highest and least active compounds from datasets $\mathrm{A}$ and $\mathrm{B}$ are shown (PBE data). Overlapped structural conformations of $\mathrm{E}_{\mathrm{x}}(\mathrm{x})$ and $\mathrm{E}_{\mathrm{x}}{ }^{+}\left(\mathrm{x}^{+}\right)$ states are shown. $E_{x}(x)$ states are shown in green carbon skeleton and $\mathrm{E}_{\mathrm{x}}{ }^{+}\left(\mathrm{x}^{+}\right)$states in magenta. (a) For $N, N$-dimethyl-p-phenylenediamine and $\mathbf{3 1}$ with small reorganization energies, only a very small change in the ring conformation was observed. (b) Notable structural changes occur in $p$-hydroxybenzoic acid and $\mathbf{3 5}$, which have large reorganization energies, particularly due to geometric changes of the carboxylate groups.

Similarly, in dataset $\mathrm{B}$, the most active compound $31\left(\mathrm{k}_{\mathrm{cat}}=45000 \mathrm{~min}^{-1}\right)$, which is orthoSH substituted thiophenol, showed a very small $\lambda(4.5-6.0 \mathrm{kcal} / \mathrm{mol}$ for three functionals) allowing a very minor geometry change upon oxidation (Figure 4a). Interestingly, the five most active compounds in this dataset $\left(\mathbf{1 0}, \mathbf{1 4}, \mathbf{2 2}, \mathbf{2 3}\right.$ and 31 with $\left.\mathrm{k}_{\mathrm{cat}}=3100-45000 \mathrm{~min}^{-1}\right)$ consistently showed small $\lambda(4.5-11.1 \mathrm{kcal} / \mathrm{mol})$ using all three functionals (Supplementary Table S10). The compounds 10, 14, 22 and 23 are ortho substituted phenols, with 10 and 14 containing one and two ortho-methoxy groups, respectively, whereas compounds 22 and 23 contain ethyl and hydroxyl groups at the ortho position. This suggests that the thiophenol with 
ortho-SH and phenols with ortho-substituted methoxy or hydroxyl groups have small reorganization energies, and accordingly are fast laccase substrates. The agreement is particularly good considering that five compounds were reported at same lowest activity due to detection limits (compounds 27, 28, 29, 34 and 35 with $\mathrm{k}_{\text {cat }} \leq 40 \mathrm{~min}^{-1}$ ), which impairs the relationship. Still, three of these compound $(\mathbf{2 8}, \mathbf{3 4}$ and $\mathbf{3 5})$ exhibited very large $\lambda(19.9-28.2$ $\mathrm{kcal} / \mathrm{mol}$ ) using all three functionals, supporting our conclusion that electronic reorganization contributes to observed turnover in real assays.

\section{Analysis of other factors contributing to activity}

Although the reorganization energy contributes to the real catalytic activity as we show here, other factors also contribute, notably the Nernst potential, the lifetime of the active poses, and the exponentially decaying electronic transmission factor ${ }^{17,23,35}$. We have studied these contributions in previous work, showing the impact of the binding affinity to the T1 site ${ }^{34}$ and the donor-acceptor distance ${ }^{23}$. Xu studied the relationship between $\log \left(\mathrm{k}_{\mathrm{cat}} / \mathrm{K}_{\mathrm{m}}\right)$ and the reduction potential differences between the $\mathrm{T} 1$ site and substrate. ${ }^{29}$ The two parameters were strongly related, although the assignment of compounds to different classes and the applied experimental potentials were collected from a range of conditions, protocols, and systems.

From the Marcus equation of the rate constant of electron transfer ${ }^{17,65}$, we expect the reorganization energy and total potential to contribute similarly. However, we expect the difference in half potentials to contribute substantially less if the assay was not performed close to standard conditions with the same concentration of T1 sites and substrates; otherwise, the reaction quotient will determine the potential more, viz. the Nernst equation. Thus, the role of the standard potential difference is less important under Michaelis-Menten assay conditions and probably in vivo. We further compared the experimental activity of datasets A and $\mathrm{B}$ to the computed Marcus activation free energy $\Delta G^{\#}=(\Delta G+\lambda)^{2} / 4 \lambda R T$ (Supplementary Figures S10 and S11). The correlations weaken relative to the reorganization energies even though the parameters are derived from the same systems and calculations; this suggests that the potential difference is less important if enzymatic turnover does not occur at standard conditions (equimolar protein $\mathrm{T} 1 /$ substrate).

Furthermore, we analyzed the correlation between the distance of the substrate-T1 site (i.e. distance between probable electron donor group of the substrate and electron acceptor residue at $\mathrm{T} 1$ site) and the $\log$ (activity) for the 15 phenolic compounds in dataset $\mathrm{A}$ (since the $\mathrm{OH}$ is the 
probable electron transfer group in phenols) docked onto the Trametes versicular laccase (PDB code: 1 GYC, data from our previous study) ${ }^{34}$ (Supplementary Figure S12). We found no correlation, which is not very surprising: Whereas a good productive conformation requires a short donor-acceptor distance as we have shown previously ${ }^{23}$, the minor variations in donoracceptor distance around this optimal value are insignificant for these similar docked phenolic compounds relative to the intrinsic differences in electronic structures. However, when the donor is not bound strongly at the T1 site, as the case for e.g. an electrode surface, minimizing the distance will obviously be very important ${ }^{14,66}$.

We note that proton transfer during the rate-limiting step in very slow substrates (large $\lambda$ ) cannot be ruled out, and occurs e.g. when the substrate is an electrode ${ }^{14}$. For the slow protein substrates the data are noisy: In dataset $\mathrm{A}^{44}$, the activity of two substrates could not detected ( $n$ propylgallate and $p$-phenylenediamine) and only four compounds, $\mathrm{p}$-hydroxybenzoic acid (3\% relative activity), pyrogallol (10\%), caffeic acid (39\%) and $o$-Cresol (39\%) showed relative activity less than ABTS (44\% activity). These substrates all exhibited high $\lambda$ values, confirming the ability of our protocol to assess these cases. Similarly, the very slow substrates of dataset B could not be accurately detected ${ }^{29}$. The $\mathrm{k}_{\text {cat }}$ of five of these compounds was $\leq 40 \mathrm{~min}^{-1}$, at the detection limit of the experiment, and three showed very high $\lambda$, as discussed above. Thus, the very slow substrates have noisy data which prevents a definite conclusion on their mechanism, including the possibility of proton-coupled electron transfer. For all other compounds than these six, our conclusions remain clear.

\section{Conclusions}

We conclude that the substrate geometric reorganization plays an important role in defining the experimentally observed catalytic activity of the laccases. We also conclude that the four-point model for computing self-exchange reorganization energies is adequate and robust to the choice of density functional. The reorganization energies of the substrates relate inversely to the enzymatic activity as expected if a larger geometric change during oxidation slows down electron transfer, and if this electron transfer step is rate-determining. In both datasets the variations in $\lambda$ can change the assayed activity by three orders of magnitude, which is thus probably a good estimate of the contribution of substrate electronic reorganization and the first such estimate of the contribution of substrate reorganization to total activity of an electron transfer protein. The fact that real activity data correlate with the substrate reorganization energy shows that this first electron-transfer step determines the ultimate turnover monitored in most laccase assays. 


\section{Supporting information}

The supplementary information file contains details on the datasets A and B (Table S1), conformation sensitivity test (Table S2), total internal energies of dataset A (Tables S3-S5) and dataset B (Table S6-S8) calculated using B3LYP, M06 and PBE functionals, reorganization energies of dataset A (Table S9) and dataset B (Table S10) calculated using total internal energies, total free energies of dataset A (Table S11-S13), total free energies of dataset B (Table S14-S16), solution phase energies of dataset A (Table S17-S19), solution phase energies of dataset B (Table S20-S22), gas phase energies of dataset A (Table S23-S25), gas phase energies of dataset B (Table S26-S28), reorganization energies calculated using free energies (Tables S29 and S30), solution phase energies (Tables S31 and S32), and gas phase energies (Tables S33 and S34), X- and Y-intersects for dataset A (Tables S35 and S36), plots of correlation between activity or $\mathrm{k}_{\mathrm{cat}}$ and reorganization energies using free energies (Figures S1 and S2), solution phase energies (Figure S3 and S4), gas phase energies (Figures S5 and S6), total internal energies (Figures S7-S9), relationship between Marcus activation barriers accounting for the oxidation potential of the substrates (Figures S10-S11), and relationship between substrate-T1 distance and $\log$ (activity) (Figure S12).

\section{Conflict of interest}

The authors declare no conflict of interest.

\section{Acknowledgement}

This study was supported by The Danish Council for Independent Research (Project ref. DFF4184-00355). 


\section{References}

1 E. I. Solomon, U. M. Sundaram and T. E. Machonkin, Chem. Rev., 1996, 96, 25632606.

2 E. I. Solomon, D. E. Heppner, E. M. Johnston, J. W. Ginsbach, J. Cirera, M. Qayyum, M. T. Kieber-Emmons, C. H. Kjaergaard, R. G. Hadt and L. Tian, Chem. Rev., 2014, 114, 3659-3853.

3 P. Giardina, V. Faraco, C. Pezzella, A. Piscitelli, S. Vanhulle and G. Sannia, Cell. Mol. Life Sci., 2010, 67, 369-385.

4 A. K. Sitarz, J. D. Mikkelsen and A. S. Meyer, Crit. Rev. Biotechnol., 2016, 36, 70-86.

5 D. K. Sandhu and D. S. Arora, Experientia, 1985, 41, 355-356.

6 D. M. Mate and M. Alcalde, Microb. Biotechnol., 2017, 10, 1457-1467.

7 U. N. Dwivedi, P. Singh, V. P. Pandey and A. Kumar, J. Mol. Catal. B Enzym., 2011, 68, 117-128.

8 A. I. Cañas and S. Camarero, Biotechnol. Adv., 2010, 28, 694-705.

9 L. Munk, M. L. Andersen and A. S. Meyer, Enzyme Microb. Technol., 2017, 106, 8896.

10 S. Rodríguez Couto and J. L. Toca Herrera, Biotechnol. Adv., 2006, 24, 500-513.

11 F. Tonin, R. Melis, A. Cordes, A. Sanchez-Amat, L. Pollegioni and E. Rosini, $N$. Biotechnol., 2016, 33, 387-398.

12 A. Kunamneni, S. Camarero, C. García-Burgos, F. J. Plou, A. Ballesteros and M. Alcalde, Microb. Cell Fact., 2008, 7, 32. http://doi.org/10.1186/1475-2859-7-32.

13 S. H. Lee, D. S. Choi, S. K. Kuk and C. B. Park, Angew. Chemie Int. Ed., 2018, 57, $7958-7985$.

14 I. Sorrentino, S. Gentil, Y. Nedellec, S. Cosnier, A. Piscitelli, P. Giardina and A. Le Goff, ChemElectroChem, 2019, 6, 1023-1027.

15 N. Hakulinen and J. Rouvinen, Cell. Mol. Life Sci., 2015, 72, 857-868.

16 K. Piontek, M. Antorini and T. Choinowski, J. Biol. Chem., 2002, 277, 37663-37669.

17 S. M. Jones and E. I. Solomon, Cell. Mol. Life Sci., 2015, 72, 869-883. 
18 A. Messerschmidt, A. Rossi, R. Ladenstein, R. Huber, M. Bolognesi, G. Gatti, A. Marchesini, R. Petruzzelli and A. Finazzi-Agró, J. Mol. Biol., 1989, 206, 513-529.

19 E. I. Solomon, D. M. Dooley, R.-H. Wang, H. B. Gray, M. Cerdonio, F. Mogno and G. L. Romani, J. Am. Chem. Soc., 1976, 98, 1029-1031.

20 L. Rulísek and U. Ryde, Coord. Chem. Rev., 2013, 257, 445-458.

21 O. Farver and I. Pecht, Mol. Cryst. Liq. Cryst., 1991, 194, 215-224.

22 C. Galli, C. Madzak, R. Vadalà, C. Jolivalt and P. Gentili, ChemBioChem, 2013, 14, 2500-2505.

23 N. J. Christensen and K. P. Kepp, J. Mol. Catal. B Enzym., 2014, 100, 68-77.

24 H. B. Gray and B. G. Malmström, Comments Inorg. Chem., 1983, 2, 203-209.

25 J. Blumberger, Chem. Rev., 2015, 115, 11191-11238.

26 H. B. Gray, B. G. Malmström and R. J. P. Williams, J. Biol. Inorg. Chem., 2000, 5, 551559 .

27 E. I. Solomon, C. H. Kjaergaard and D. E. Heppner, in Electrochemical Processes in Biological Systems, John Wiley \& Sons, Inc Hoboken, NJ, 2015, pp. 169-212.

28 C. Stines-Chaumeil, E. Roussarie and N. Mano, Biochim. open, 2017, 4, 36-40.

29 F. Xu, Biochemistry, 1996, 35, 7608-7614.

30 O. V Morozova, G. P. Shumakovich, M. A. Gorbacheva, S. V Shleev and A. I. Yaropolov, Biochem., 2007, 72, 1136-1150.

31 M. Frasconi, G. Favero, H. Boer, A. Koivula and F. Mazzei, Biochim. Biophys. Acta, 2010, 1804, 899-908.

32 D. M. Mate and M. Alcalde, Biotechnol. Adv., 2015, 33, 25-40.

33 G. Macellaro, M. C. Baratto, A. Piscitelli, C. Pezzella, F. Fabrizi De Biani, A. Palmese, F. Piumi, E. Record, R. Basosi and G. Sannia, Appl. Microbiol. Biotechnol., 2014, 98, 4949-4961.

34 R. Mehra, J. Muschiol, A. S. Meyer and K. P. Kepp, Sci. Rep., 2018, 8, 17285.

35 R. Mehra, A. S. Meyer and K. P. Kepp, RSC Adv., 2018, 8, 36915-36926. 
L. Hu, M. Farrokhnia, J. Heimdal, S. Shleev, L. Rulíšek and U. Ryde, J. Phys. Chem. B, 2011, 115, 13111-13126.

J. Li, M. Farrokhnia, L. Rulíšek and U. Ryde, J. Phys. Chem. B, 2015, 119, 8268-8284.

K. P. Kepp, Inorg. Chem., 2015, 54, 476-483.

39 Y. Zhao, S. Bhattacharjee, B. M. Jones, J. M. Hill, C. Clement, K. Sambamurti, P. Dua and W. J. Lukiw, Mol. Neurobiol. Mol. Neurobiol, Mol Neurob, Mol Neurobiol, Mol. Neurobiol. a Rev. J., 2015, 52, 533-544.

40 A. J. Di Bilio, M. G. Hill, N. Bonander, B. G. Karlsson, R. M. Villahermosa, B. G. Malmström, J. R. Winkler and H. B. Gray, J. Am. Chem. Soc., 1997, 119, 9921-9922.

41 H. J. Wijma, I. MacPherson, O. Farver, E. I. Tocheva, I. Pecht, M. P. Verbeet, M. E. P. Murphy and G. W. Canters, J. Am. Chem. Soc., 2007, 129, 519-525.

42 M. T. Nielsen, K. A. Moltved and K. P. Kepp, Inorg. Chem., 2018, 57, 7914-7924.

43 M. H. M. Olsson, U. Ryde and B. O. Roos, Protein Sci., 1998, 7, 2659-2668.

44 W. T. Sulistyaningdyah, J. Ogawa, H. Tanaka, C. Maeda and S. Shimizu, FEMS Microbiol. Lett., 2004, 230, 209-214.

45 S. F. Nelsen, S. C. Blackstock and Y. Kim, J. Am. Chem. Soc., 1987, 109, 677-682.

46 LigPrep, Schrödinger, LLC, New York, NY, 2017.

47 Jaguar, Schrödinger, LLC, New York, NY, 2017.

48 A. D. Bochevarov, E. Harder, T. F. Hughes, J. R. Greenwood, D. A. Braden, D. M. Philipp, D. Rinaldo, M. D. Halls, J. Zhang and R. A. Friesner, Int. J. Quantum Chem., 2013, 113, 2110-2142.

49 P. Pulay, J. Comput. Chem., 1982, 3, 556-560.

50 Ö. Farkas, Ö. Farkas, Ö. Farkas and H. B. Schlegel, Phys. Chem. Chem. Phys., 2002, 4, $11-15$.

51 P. J. Stephens, F. J. Devlin, C. F. Chabalowski and M. J. Frisch, J. Phys. Chem., 1994, 98, 11623-11627.

52 A. D. Becke, J. Chem. Phys., 1993, 98, 1372-1377.

53 C. Lee, W. Yang and R. G. Parr, Phys. Rev. B, 1988, 37, 785-789. 
54 Y. Zhao and D. G. Truhlar, Theor. Chem. Acc., 2008, 120, 215-241.

55 J. P. Perdew, K. Burke and M. Ernzerhof, Phys. Rev. Lett., 1996, 77, 3865.

56 K. P. Jensen, J. Inorg. Biochem., 2008, 102, 87-100.

57 K. P. Jensen, J. Inorg. Biochem., 2006, 100, 1436-1439.

58 S. F. Nelsen and M. J. R. Yunta, J. Phys. Org. Chem., 1994, 7, 55-62.

59 T. A. Halgren, J. Chem. Inf. Model., 2009, 49, 377-389.

60 R. A. Friesner, J. L. Banks, R. B. Murphy, T. A. Halgren, J. J. Klicic, D. T. Mainz, M. P. Repasky, E. H. Knoll, M. Shelley, J. K. Perry, D. E. Shaw, P. Francis and P. S. Shenkin, J. Med. Chem., 2004, 47, 1739-1749.

61 R. A. Friesner, R. B. Murphy, M. P. Repasky, L. L. Frye, J. R. Greenwood, T. A. Halgren, P. C. Sanschagrin and D. T. Mainz, J. Med. Chem., 2006, 49, 6177-6196.

62 O. Salomon, M. Reiher and B. A. Hess, J. Chem. Phys., 2002, 117, 4729-4737.

63 K. P. Kepp, Coord. Chem. Rev., 2013, 257, 196-209.

64 F. Neese, J. Biol. Inorg. Chem., 2006, 11, 702-711.

65 R. A. Marcus and N. Sutin, Biochim. Biophys. Acta (BBA)-Reviews Bioenerg., 1985, 811, 265-322.

66 O. Schlesinger, M. Pasi, R. Dandela, M. M. Meijler and L. Alfonta, Phys. Chem. Chem. Phys., 2018, 20, 6159-6166. 